\title{
Efficiency And Impact Factors of Anatomical Intelligence For Breast And Hand-Held Ultrasound In Lesion Screening: A Pilot Study
}

\section{Chaochao Dai}

Zhejiang University School of Medicine

Lingyun Bao ( $\nabla_{\text {jttj571@sina.com ) }}$

Zhejiang University School of Medicine

Hongju Yan

Zhejiang University School of Medicine

Luoxi Zhu

Zhejiang University School of Medicine

\section{Xiaojing Xu}

Zhejiang University School of Medicine

\section{Yanjuan Tan}

Zhejiang University School of Medicine

\section{Lifang Yu}

Zhejiang University School of Medicine

\section{Jing Yang}

Zhejiang University School of Medicine

Chenxiang Jiang

Zhejiang University School of Medicine

\section{Yingzhao Shen}

Zhejiang University School of Medicine

\section{Research Article}

Keywords: Breast, Ultrasound, Anatomical intelligence for breast

Posted Date: January 24th, 2022

DOI: https://doi.org/10.21203/rs.3.rs-1172382/v1

License: (c) (1) This work is licensed under a Creative Commons Attribution 4.0 International License.

Read Full License 


\section{Abstract}

Objective: To investigate the efficiency and impact factors of anatomical intelligence for breast (AlBreast) and hand-held ultrasound (HHUS) in lesion screening.

Methods: A total of 172 outpatient women were randomly selected, underwent Al-Breast ultrasound (Group Al) once and HHUS twice. HHUS was performed by breast image radiologists (Group A) and general radiologists (Group B). For the Al-Breast examination, a trained technician performed the wholebreast scan and data acquisition, while other general radiologists performed image interpretation. The examination time and lesion detection rate were recorded. The impact factors for breast lesion screening, including breast cup size, lesion number (LN), and benign or malignant lesions were analyzed.

Results: Scan times of Groups Al, A, and B were $262.15 \pm 40.4$ s, $237.5 \pm 110.3 \mathrm{~s}, 281.2 \pm 86.1$ s, respectively. The scan time of Group Al was significantly higher than Group $\mathrm{A}(P<0.01)$, but was slightly lower than Group B ( $P>0.05)$. The detection rates of Group Al, A, and B were $92.8 \pm 17.0 \%, 95.0 \pm 13.6 \%$, and $85.0 \pm 22.9 \%$, respectively. Comparable lesion detection rates were observed in Group Al and Group A $(P>0.05)$, but a significantly lower lesion detection rate was observed in Group B compared to the other two (both $P<0.05$ ). Regarding missed diagnosis rates of malignant lesions, comparable performance was observed in Group Al, Group A, and Group B ( $8 \%$ vs. $4 \%$ vs. $14 \%$, all $P>0.05$ ). We found a strong linear correlation between scan time and cup size in Group $\mathrm{Al}(\mathrm{r}=0.745)$. No impacts of cup size and $\mathrm{LN}$ were found on the lesion detection rate in Group Al ( $P>0.05)$.

Conclusions: The screening efficiency of Al-Breast ultrasound was comparable to that of a breast image radiologist and superior to that of the general radiologist. Al-Breast ultrasound may be used as a potential approach for breast cancer screening.

\section{Introduction}

Breast cancer is the leading malignant tumor to severely threat female health worldwide, accounting for $11.7 \%$ of the new cases of cancer in 2020. In particular, mortality rates are higher in developing countries [1]. Early detection and diagnosis of breast cancer is critical, so standardized screening is of great importance. Asian women have relatively small and dense breasts; in China, half of those aged 45-65 are classified as dense type [2, 3]. This category has a 4-6 folds increased risk of breast cancer than women with non-dense breasts. Mammography, a classic screening tool, has limitations on dense breasts; thus, screening sensitivity is reduced in China [4]. Moreover, according to the national registry, the breast cancer detection rate is less than 1\% [5]. In the past 20 years, the prevalence of this disease has increased by 3$5 \%$ every year, much faster than the average increase rate of $0.5 \%$ worldwide [6].

Conventional hand-held ultrasound (HHUS) is a preferred approach for the screening of breast cancer in Chinese women [7]. The accuracy and efficiency of the screening is related to the experience of the radiologist $[8,9]$. On the other hand, anatomical intelligence for breast (Al-Breast) is a new ultrasound method [10] that makes the scan process visual. Al-Breast uses a magnetic positioning device and 
separates real-time scan and interpretation diagnosis. This study aimed to explore the value of Al-Breast in breast lesion detection and the possibility of its use for breast cancer screening, while comparing it to HHUS.

\section{Materials And Methods}

A single-center study was carried out in the Affiliated Hangzhou First People's Hospital, School of Medicine, Zhejiang University, from February to April 2021.The project was approved by the Ethics

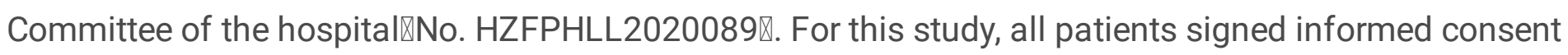
and accepted the use of their image data.

\section{Study participants and study design}

A total of 172 female patients, aged 18-80 years, were enrolled at the Breast Department. They presented breast pain, lumps (initial diagnosis or reexamination), nipple discharge, or abnormal imaging findings. Patients who met the inclusion criteria were 1) about to undergo surgery or needle biopsy for breast lesions; 2) Included in a long-term follow-up for breast lesions ( $\geq 2$ years) and had complete data. The exclusion criteria listed those with cardiac pacemaker implantation, previous total mastectomy, breast implantation, or in lactation and pregnancy.

All patients received Al-Breast ultrasound scan once and HHUS twice. The Al-Breast ultrasound group (Group Al) included two general radiologists that interpreted the images (5-8 years of experience in ultrasound diagnosis, C.C, and H.J) and one technician (short training in breast scan).

HHUS was performed by breast image radiologists (Group A) or general radiologists (Group B). The radiologists in Group A had 8-10 years of experience in breast ultrasound (X.X and Y.J) while those in Group B had a 5 to 8-year expertise in ultrasound diagnosis (L.F and J.J).

\section{Al-Breast ultrasound}

A color ultrasound instrument (Philips EPIQ5) equipped with a variable-frequency probe (eL18-4) was used. This technology comprised a frequency range of 2-22 MHz, a receivable magnetic field signal, and a Al-Breast magnetic positioning device mattress.

In Group Al, the technician conducted the scanning as follows: the patient took a supine position with arms raised above the head, and the targeted breast was raised by appropriate lateral rotation. Triangular support behind the examined side helped fix the position and place the breast into an effective magnetic field. The scan area was determined through locating the nipple and the lower, inner, and outer margin of the breast. To ensure clearness in the images, a relatively uniform and alternating up-down scan mode was selected, with at least a 1/3 overlap between every two scans. A bilateral double breast examination was performed. During the exploration, the breast surface marks were filled with green thick lines. Complete filling meant that the examination of a unilateral breast was finished. The technician in the 
Group Al performed a whole-breast scanning according to the standards and recorded the time of scanning completion.

Subsequently, the breast data were saved in video by the technician, imported into a computer, and interpreted using professional software by the radiologists. The time of interpretation, and lesion number (LN) and location were recorded.

\section{HHUS examination}

The previously described Philips EPIQ5 instrument equipped with the eL18-4 probe was employed. Examinations in Groups $A$ and $B$ began with patients taking a supine position and fully exposing their chest. A comprehensive breast scanning was performed radially or alternating up and down, left and right. During the examination, the images were saved and the number and specific location of the lesions were recorded.

\section{Number and categorization of breast lesions}

Breast lesion detection and patient clinical data were analyzed in the three groups. If the LN was the same in the three groups, there was no controversy. If the LN was different, two radiologists in Group A reviewed the Al-Breast image information and saved $2 \mathrm{D}$ ultrasound images to make a judgment. If necessary, patients were called for reexamination.

Criteria for benign and malignant breast lesions: 1) lesions with BI-RADS categories 4 and above were confirmed as benign or malignant by surgery or puncture; 2) lesions with BI-RADS 3 and below were confirmed by surgery. In the cases were no surgery nor puncture was conducted, all lesions were followed up for at least 2 years ( $\geq 2$ times). Ultrasound images of the last 2 years were reviewed. No significant change in the size and ultrasound characteristics of the lesions was judged benign. Lastly, $10 \%$ of patients were randomly selected for MRI to exclude false positives.

\section{Statistical analysis}

The MedCal software was used for statistical analyses. Measurement data were expressed as mean \pm SD. Differences in measurement data between two groups were analyzed by the Mann-Whitney test, with $P<0.05$ termed as statistical significance. Differences in measurement data between multiple groups were analyzed by the Anova test, with $P \otimes 0.05$ indicating a significant difference between at least two groups. Further evaluations included the use of Spearman's correlation, with $|P|=0.7,0.7>|P|>0.4$,and $|P|<0.4$ indicating high, weak and no significant linear correlation, respectively.

\section{Results}

Participant characteristics 
A total of 172 patients were enrolled, with an average age of 41.3 years old (SD \pm 13.3 ). Breast cups $A, B$, $C$, and $D$ and above accounted for $26 \%, 40 \%, 27 \%$, and $7 \%$, respectively. $L N$ values of $0,1,2$, and 3 and more were distributed as present in the $8 \%, 23 \%, 19 \%$, and $50 \%$, respectively, of the patients (Table 1 ).

In this study, 15 cases had no lesions confirmed by ultrasound during the follow-up, while the remaining 157 women had a total of 555 lesions. Of them, 132 women underwent surgery or puncture and counted a total of 163 lesions that were: 1 ) malignant $(\mathrm{N}=51)$ : 36 lesions of invasive breast carcinoma (nonspecial type, NST), 6 lesions of ductal carcinoma in situ(DCIS), 3 lesions of mucinous carcinoma, 5 lesions of papillary carcinoma, and 1 lesion of adenoid cystic carcinoma; and 2$)$ benign $(N=112): 81$ lesions of fibroadenomas, 4 lesions of inflammatory lesions, 6 lesions of intraductal papillomas, 18 lesions of adenopathy, 1 lesion of lipoma and 2 lesions of fat calcified nodules. There were 392 lesions without pathological results. Of them, 70 lesions were confirmed as benign by MRI. The remaining 322 lesions were confirmed as benign by ultrasound in the follow-up (Table 1).

Table 1

Basic characteristics of enrolled patients

\begin{tabular}{|llll|}
\hline Group & Enrolled cases (n=172) & Benign (n=128) & Malignant (n=44) \\
\hline Lesion number & & & \\
\hline 0 & $15(8 \%)$ & $15(12 \%)$ & $0(0 \%)$ \\
\hline 1 & $41(23 \%)$ & $18(14 \%)$ & $23(52 \%)$ \\
\hline 3 & $32(19 \%)$ & $23(18 \%)$ & $9(20 \%)$ \\
\hline 4 & $28(16 \%)$ & $22(17 \%)$ & $6(14 \%)$ \\
\hline Breast cup size & & $15(12 \%)$ & $4(9 \%)$ \\
\hline A & $19(11 \%)$ & $35(27 \%)$ & $2(5 \%)$ \\
\hline B & $44(26 \%)$ & & $12(27 \%)$ \\
\hline C & $69(40 \%)$ & $32(25 \%)$ & $15(34 \%)$ \\
\hline D and above & $12(7 \%)$ & $54(42 \%)$ & $13(30 \%)$ \\
\hline
\end{tabular}

Examination time with Al-Breast and HHUS

Scan times of Groups A, B, and Al were 237.5 $\pm 110.3 \mathrm{~s}\left(\mathrm{~T}_{\mathrm{A}}\right), 281.2 \pm 86.1 \mathrm{~s}\left(\mathrm{~T}_{\mathrm{B}}\right)$, and $262.15 \pm 40.4 \mathrm{~s}\left(\mathrm{~T}_{\mathrm{Al}}\right.$ scan $)$, respectively. Group $A I$ interpretation time was $225.6 \pm 43.6 \mathrm{~s}\left(T_{A l}\right.$ interpretation $) . T_{A}$ was significantly shorter than $\mathrm{T}_{\mathrm{B}}$ and $\mathrm{T}_{\mathrm{Al}}$ scan $($ Both $P \otimes 0.05)$, and $\mathrm{T}_{\mathrm{Al}}$ scan was relatively shorter than $\mathrm{T}_{\mathrm{B}}(P=0.057)$ (Fig. 1). 
In the non-lesion groups, the scan times were $173.7 \pm 34.9 \mathrm{~s}\left(\mathrm{~T}_{\mathrm{A}}\right), 217.5 \pm 50.6 \mathrm{~s}\left(\mathrm{~T}_{\mathrm{B}}\right)$, and $239.5 \pm 30.5 \mathrm{~s}\left(\mathrm{~T}_{\mathrm{Al}}\right.$ scan), while $T_{A l}$ interpretation was $193.7 \pm 34.1 \mathrm{~s}$. For those with $L N 1-5$, times were $223.5 \pm 75.9 \mathrm{~s}\left(\mathrm{~T}_{\mathrm{A}}\right)$, $275.8 \pm 77.4 \mathrm{~s}\left(\mathrm{~T}_{\mathrm{B}}\right), 259.8 \pm 35.4 \mathrm{~s}\left(\mathrm{~T}_{\mathrm{Al}}\right.$ scan $)$, and $223.7 \pm 38.8 \mathrm{~s}\left(\mathrm{~T}_{\text {Al interpretation }}\right)$. In the $\mathrm{LN} \varangle 5$ cases, times were $340.6 \pm 190.9 \mathrm{~s}\left(\mathrm{~T}_{A}\right), 342.6 \pm 106.1 \mathrm{~s}\left(\mathrm{~T}_{B}\right), 286.3 \pm 55.7 \mathrm{~s}\left(\mathrm{~T}_{\mathrm{Al}}\right.$ scan $)$, and $251.1 \pm 56.1 \mathrm{~s}\left(\mathrm{~T}_{\text {Al interpretation }}\right)$. The scan times of patients with lesions were significantly longer than those of patients without lesions. In particular, Groups A and B had a positive linear correlation between the scan time and the $L N\left(r_{a}=0.512\right.$, $\left.r_{b}=0.397\right)$. The scan time in Group Al did not correlate with $L N\left(r_{A l}\right.$ scan $\left.=0.156\right)$. When $L N$ was $1-5$, there was no statistical significance ( $P>0.05)$ (Table 2 and 4$)$.

Furthermore, scan times significantly increased with breast size in the three groups. In particular, $\mathrm{T}_{\mathrm{Al}}$ scan had a positive linear correlation with breast size $\left(r_{A l}\right.$ scan $\left.=0.745\right)$ (Table 2 and 4). On the other hand, the scan times of benign cases from Group A and group B were significantly longer than those of malignant cases $(P<0.05) . \mathrm{T}_{\mathrm{Al}}$ scan did not correlate with benign or malignant lesions, and there were no significant differences $(P=0.688)$ (Table 2 and 4$)$.

Lesion detection rate with Al-Breast and HHUS

In this study, the average detection rates of breast lesions were: Group A (95.0 $\pm 13.6 \%)$, Group Al $(92.8 \pm 17.0 \%)$ and Group B (85.0 $\pm 22.9 \%)$. No statistical significance was noted between Group Al and Group A ( $P=0.367)$. However, differences were found when comparing Group A and Group Al, respectively, with Group B (both $P<0.05)$.

Detection rates in Group A and Group Al did not correlate with $L N$, and there was no statistical significance. For its part, the detection rate in Group $B$ had a negative linear correlation with the $L N\left(r_{b}=\right.$ -0.436, $P \otimes 0.001$ ). In Group $B$, patients with $L N \geq 2$ and $C$ cup and above had a decreased detection rate that was significantly lower than those in Group A and Group AI $(P<0.05)$ (Table 3 and 4). Moreover, detection rates throughout the three groups did not correlate with breast size ( $P>0.05)$ (Table 3 and 4$)$.

For benign lesions, missed diagnosis rates in Group A, Group Al, and Group B were 7\%, $8 \%$, and 20\%, respectively. Group B had higher rates than Group A and Group Al (both $P<0.05$ ) while there were no statistical differences between Group Al and Group A $(P=0.629)$. For malignant lesions, missed diagnosis rates in Group A, Group Al, and Group B were $4 \%, 8 \%$, and $14 \%$, respectively. There were no statistical differences between them $(P>0.05)$ (Table 5). 
Table 2

Impact of LN, breast size, and pathological properties on the examination time in the three groups

\section{Group}

$\otimes 5$

P-value

1

2

3

4

5

P-value

Breast cup size

A

B

C

D and above

P-value

Pathological properties

Number of malignant lesions $(\mathrm{n}=44)$

Number of benign lesions $(n=113)$

P-value
HHUS

$\mathrm{T}_{\mathrm{A}}(\mathbf{s})$

$\mathrm{T}_{\mathrm{B}}(\mathbf{s})$

$173.7 \pm 34.9$

$217.5 \pm 50.6$

$223.5 \pm 75.9$

$275.8 \pm 77.4$

$340.6 \pm 190.9$

0.027

0.038

0.688

0.770

Al-Breast

$\mathrm{T}_{\mathrm{Al} \text { scan }}(\mathbf{s}) \quad \mathrm{T}_{\mathrm{Al} \text { interpretation }}(\mathbf{s})$

$239.5 \pm 30.5 \quad 193.7 \pm 34.1$

$259.8 \pm 35.4 \quad 223.9 \pm 38.8$

$286.3 \pm 55.7 \quad 251.1 \pm 56.1$

$<0.001<0.001<0.001<0.001$

$183.5 \pm 61.7 \quad 254.4 \pm 63.7 \quad 265.9 \pm 37.5 \quad 228.1 \pm 37.9$

$210.9 \pm 67.7 \quad 254.0 \pm 69.3 \quad 254.6 \pm 35.0 \quad 220.3 \pm 37.0$

$258.8 \pm 71.9 \quad 296.4 \pm 80.6 \quad 262.1 \pm 35.4 \quad 225.1 \pm 44.2$

$248.1 \pm 84.2 \quad 295.0 \pm 81.1 \quad 250.0 \pm 27.4 \quad 215.8 \pm 34.7$

$282.2 \pm 59.9 \quad 338.9 \pm 91.8 \quad 263.0 \pm 42.0 \quad 230.6 \pm 41.0$

$<0.001$

0.003

0.474

0.758

$205.0 \pm 84.1 \quad 248.3 \pm 71.2 \quad 230.6 \pm 20.3 \quad 193.7 \pm 25.0$

$228.4 \pm 75.3 \quad 284.7 \pm 88.6 \quad 250.1 \pm 20.8 \quad 215.2 \pm 26.8$

$231.9 \pm 72.2 \quad 293.6 \pm 89.1 \quad 286.8 \pm 27.8 \quad 248.8 \pm 35.8$

$431.8 \pm 242.1 \quad 332.8 \pm 76.1 \quad 350.4 \pm 43.9 \quad 311.3 \pm 47.9$

$<0.001$

0.007

$<0.001$

$<0.001$

$220.0 \pm 122.4 \quad 252.4 \pm 64.5 \quad 266.0 \pm 42.6 \quad 228.8 \pm 43.3$

$243.6 \pm 105.7 \quad 291.1 \pm 90.5 \quad 260.9 \pm 39.8 \quad 224.5 \pm 43.8$ 
Table 3

Impact of LN and breast size on the detection rates in the three groups

HHUS

Group A Group B P
Group Al Comparison of P-value between Al and HHUS

Group A
Group B

\section{Lesion}

Number

\begin{tabular}{|lllllll|}
\hline & $100.0 \pm 0.0$ & $100.0 \pm 0.0$ & 1.0000 & $100.0 \pm 0.0$ & 1.0000 & 1.0000 \\
\hline 1 & $100.0 \pm 0.0$ & $95.1 \pm 21.8$ & 0.7038 & $95.1 \pm 21.8$ & 0.7038 & 1.0000 \\
\hline $2 \leq \mathrm{LN} \leq 3$ & $92.5 \pm 79.8$ & $79.8 \pm 26.2$ & 0.0152 & $90.6 \pm 17.7$ & 0.5816 & 0.0450 \\
$\geq 4$ & $92.6 \pm 14.4$ & $79.1 \pm 18.3$ & 0.0001 & $91.5 \pm 13.8$ & 0.5924 & 0.0002 \\
\hline P-value & 0.054 & $<0.001$ & NA & 0.385 & NA & NA \\
$\begin{array}{l}\text { Breast } \\
\text { size }\end{array}$ & & & & & & \\
\hline A & $93.5 \pm 14.0$ & $86.1 \pm 20.7$ & 0.1572 & $93.8 \pm 14.0$ & 0.8806 & 0.1247 \\
B & $95.9 \pm 13.7$ & $87.8 \pm 20.9$ & 0.0216 & $94.3 \pm 15.4$ & 0.4970 & 0.0812 \\
\hline C & $93.8 \pm 14.5$ & $82.8 \pm 24.5$ & 0.0031 & $88.2 \pm 22.0$ & 0.3260 & 0.0461 \\
$\begin{array}{l}\text { D and } \\
\text { above }\end{array}$ & $100.0 \pm 0.0$ & $73.3 \pm 31.9$ & 0.0153 & $98.5 \pm 5.2$ & 0.7290 & 0.0262 \\
\hline P value & 0.416 & 0.197 & NA & 0.193 & NA & NA \\
\hline
\end{tabular}


Table 4

Correlation between $\mathrm{LN}$, breast size, examination time, and detection rate in the three groups

\begin{tabular}{|lll|}
\hline & Lesion Number & Breast size \\
\hline Examination time & & \\
\hline Scan time of Group Al & $r=0.156(P=0.042)$ & $r=0.745(P<0.001)$ \\
\hline Interpretation time of Group Al & $r=0.195(P=0.011)$ & $r=0.665(P<0.001)$ \\
\hline Group A & $r=0.512(P<0.001)$ & $r=0.267(P<0.001)$ \\
\hline Group B & $r=0.397(P<0.001)$ & $r=0.268(P<0.001)$ \\
Detection rate & & \\
\hline Group Al & $r=-0.248(P=0.001)$ & $r=-0.0756(P=0.218)$ \\
\hline Group A & $r=-0.275(P<0.001)$ & $r=-0.0943(P=0.536)$ \\
Group B & $r=-0.436(P<0.001)$ & $r=-0.0473(P=0.218)$ \\
\hline
\end{tabular}

Table 5

Influence of benign and malignant lesions on the examination time and missed detection rate in the three groups

\begin{tabular}{|c|c|c|c|c|c|c|}
\hline & \multicolumn{3}{|l|}{ HHUS } & \multirow[t]{2}{*}{$\begin{array}{l}\text { Group } \\
\text { Al }\end{array}$} & \multicolumn{2}{|c|}{$\begin{array}{l}\text { Comparison of P-value } \\
\text { between Al and HHUS }\end{array}$} \\
\hline & $\begin{array}{l}\text { Group } \\
\text { A }\end{array}$ & Group B & $\mathbf{P}$ & & Group A & Group B \\
\hline \multicolumn{7}{|l|}{ Missed diagnosis rate (LN) } \\
\hline $\begin{array}{l}\text { Number of benign lesions } \\
(n=504)\end{array}$ & $35(7 \%)$ & $102(20 \%)$ & $<.001$ & $40(8 \%)$ & 0.6290 & $<0.001$ \\
\hline $\begin{array}{l}\text { Number of malignant } \\
\text { lesions }(n=51)\end{array}$ & $2(4 \%)$ & $7(14 \%)$ & 0.156 & $4(8 \%)$ & 0.6646 & 0.515 \\
\hline Chi-square test $P$ & 0.186 & 0.398 & NA & 0.787 & NA & NA \\
\hline
\end{tabular}

\section{Discussion}

Mammography is considered the main imaging method for the reduction of breast cancer mortality. However, the sensitivity of this method is lower in dense breasts. In China and other Asian countries, the proportion of women with dense breasts is higher than in western countries. Ultrasound systems overcome this issue with high screening efficiency in dense breasts [11,12]. Because of this, the ultrasound is the main imaging method for breast cancer screening or diagnosis in China and the main supplementary method for screening in other countries. Nevertheless, the reliability of the conventional 
HHUS is still controversial due to high false-positive rates and significant operator dependence [13-15]. In Filip Šroubek et al whole-breast ultrasound study, about 3\% of the cases missed at least a portion of 1 $\mathrm{cm}^{2}$ [16]. Another issue is that the central parts of the breast have better coverage compared to the periphery. Meanwhile, the poor repeatability of conventional HHUS reduces the accuracy of breast lesions follow-up $[14,17,18]$. In this present work, we focused on how to solve the ultrasound operator dependence and to reduce the risk of missed breast lesions. We also discuss some new technologies that have been developed and applied in the clinic.

A standardized breast scan is central to solve missed diagnoses of breast lesions. The current automated breast ultrasound applies wide-amplitude high-frequency probes to achieve a standardized scan. Previous studies have confirmed that the automated breast ultrasound does not depend on the operator's experience $[6,17]$ and increases the sensitivity and specificity of breast cancer screening when compared with mammography. The whole breast ultrasound (WBUS) system based on electromagnetic tracking has been reported as less dependent on the operator than HHUS $[14,19]$. For its part, the Al-Breast ultrasound is similar to WBUS. Visualization during the whole scan process works through breast region localization and magnetic navigation, ensuring that the scan covers the whole breast and reduces blind areas. Moreover, the scan can be carried out according to a set standardized workflow, to solve the same HHUS problems. Meanwhile, the breast information is comprehensively recorded in video. The subject suffers no discomfort. Applying a standard examination can improve the accuracy and repeatability of breast screening [14]. Moreover, Al-Breast technicians are trained in a short time; then they can finish the whole breast scan independently and ensure the clearness of images for interpretation. With respect to radiologists, they do not need extra long-time training or accumulation of image interpretation experience to improve the efficiency of image interpretation.

This study compared the screening efficiency of both the method (Al-Breast ultrasound and HHUS) and the operator (radiologists with different years of experience) in breast lesions under a daily workflow mode. There have been many reports on the detection rate of breast cancer by HHUS. According to Lin X et al.'s study [6], the detection rate is up to $96 \%$, but that of Niu $L$ et al.'s [20] claims it is only $82 \%$. A metaanalysis by Zhang $X$ et al. [21] revealed that HHUS produces a $90 \%$ rate with a large deviation that is closely related to the operator's diagnostic experience. In this study, we found that the Al-breast ultrasound reported a higher breast lesions rate than that conducted by general radiologists. The results also showed that it was equivalent to breast image radiologists. The corresponding missed diagnosis rates of breast cancer in Group Al, Group A, and Group B were 8\%, 4\%, and 14\%, respectively. Although there was no statistical significance, the Al-Breast ultrasound reported low missed diagnosis of malignant lesions and high detection rates, which are of great importance in the clinic. For breast cancer patients, early detection and diagnosis can improve the survival rate.

It has been reported that the lesion detection rate of HHUS may correlate with breast size, lesion size, and LN [22-26]. The smaller the breast lesion is, the lower the detection rate. When the breast size is large, the lesions tend to locate in the deep tissue, which increases the difficulty in the ultrasound analysis and reduces the detection rate. In this study, the detection rate of the Al-breast ultrasound was not affected by 
breast size, LN, benign or malignant lesions, consistent with breast image radiologists. However, there was a negative linear correlation between the detection rate of general radiologists and LN. Additionally, their detection rate was significantly lower than that of Al-breast ultrasound in the cases with $L N \geq 2$ and C cup or above. More than 1/3 (22/60) of the breast lesions missed by general radiologists were of less than $1 \mathrm{~cm}$; the small size was probably the major reason for a low detection rate. On the other hand, lesions missed by the latter operators were found in the periphery of the breasts; thus, incomplete coverage could be an important factor leading to an unsuccessful diagnosis. The detection rate of the AlBreast ultrasound is relatively higher compared with that periphery situation, indicating that this standardized scan is able to avoid insufficient coverage and reduce missed diagnosis.

In the efficiency assessment of breast cancer screening, the most important indicator is the detection rate of breast lesions, followed by scan time. In this study, the scan time was significantly influenced by breast size, and it increased with cup size in the three groups. The scan time of the Al-breast ultrasound had a positive linear correlation with breast size at a relatively constant time for different breast cups. In the HHUS group, the scan time of radiologists was also impacted by $L N$, with a positive linear correlation between them in both groups. Al-Breast ultrasound was the least affected by LN. Also, there was no correlation between scan time and LN (1-5). However, the Al-Breast ultrasound successively extended the

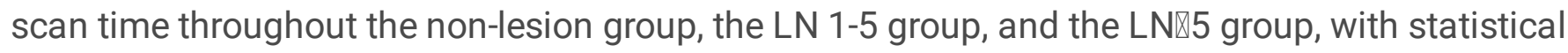
differences among the three. The reason for this could be the proportion of patients with large breasts (patients with C Cup and above): in the non-lesion group, they accounted for $27 \%$ of the patients (4/15); in the LN 1-5 group, 34\% (44/130); and in the LN $₫ 5$ group, $41 \%(11 / 27)$. When the proportion of patients with large breasts increased, the average scan time would prolong. In terms of scan time alone, the time spent by radiologists was the shortest, while that of Al-Breast was relatively shorter than general radiologists. Nevertheless, due to the different characteristics of scan and diagnosis in Al-Breast ultrasound, extra interpretation time was needed; therefore, the whole examination time was longer than those of the other two groups.

This study has some limitations as a single-center project with data from a hospital. The radiologists had relatively wide experience in breast diagnosis; thus the Al-Breast ultrasound had a high detection rate of breast lesions. Multicenter and large-sample databases are needed to assess the efficiency of the AlBreast ultrasound in asymptomatic patients and primary hospital screening. Indeed, electromagnetic tracking devices have their limitations. An electromagnetic generator is usually placed near the head of the scan bed, and the magnetic sensor has a limited detection range. Thus, the patient needs to be close to the generator as much as possible. Simultaneously, patients need to remove metal and electronic objects from their bodies before the scan to prevent interference in the magnetic field.

\section{Conclusion}

Above all, Al-Breast ultrasound is similar to the conventional HHUS but achieves a separation between diagnosis and scan. Image scan is standardized, reproducible, and provides more objective location information in the daily clinical practice. This grants a research basis for the remote consultation and 
breast cancer screening. The efficiency of the Al-Breast ultrasound screening is equivalent to the breast image radiologist. Its advantages, such as improving operator dependency, increasing the screening sensitivity, technician training with less time and cost, and focusing breast diagnosis to radiologists, have better social and economic benefits. Al-Breast ultrasound may be a potential approach for breast cancer screening in primary hospitals.

\section{Declarations}

\section{Ethics approval and consent to participate}

This study was approved by the Institutional Review Board and Ethics Committee of Affiliated Hangzhou First People's Hospital,Zhejiang University School of Medicine. All patients provided signed informed consent before the examination. All methods were performed in accordance with the relevant guidelines and regulations.

\section{Consent for publication}

Not applicable.

\section{Availability of data and materials}

The authors confirm that the data supporting the findings of this study are available within the article.

\section{Competing interests}

The authors declare that there is no confict of interest regarding the publication of this paper.

\section{Funding}

This work is supported by the Construction Fund of Medical Key Disciplines of Hangzhou.

\section{Authors' contributions}

LB designed and organized this study; $C D, H Y$ and $L Z, X X$ summarized and analyzed the multicenter data and wrote the manuscript; YT,LY,JY and CJ,YS prepared and recorded the data. All authors reviewed the manuscript.

\section{Acknowledgments}

The authors would like to express their gratitude to MedSci (https://editing.medscihealthcare.com/dist/medsci-order/\#/verify) for the expert linguistic services provided.

\section{References}


1. Sung H, Ferlay J, Siegel RL, et al. Global Cancer Statistics 2020: GLOBOCAN Estimates of Incidence and Mortality Worldwide for 36 Cancers in 185 Countries. CA Cancer J Clin. 2021;71:209-249.

2. Dai $\mathrm{H}$, Yan $\mathrm{Y}$, Wang $\mathrm{P}$, et al. Distribution of mammographic density and its influential factors among Chinese women. Int J Epidemiol. 2014;43:1240-51.

3. Liu J, Liu PF, Li JN, et al. Analysis of mammographic breast density in a group of screening chinese women and breast cancer patients. Asian Pac J Cancer Prev. 2014;15:6411-4.

4. Boyd NF, Guo H, Martin LJ, et al. Mammographic density and the risk and detection of breast cancer. N Engl J Med. 2007;356:227-36.

5. Zeng H, Chen W, Zheng R, et al. Changing cancer survival in China during 2003-15: a pooled analysis of 17 population-based cancer registries. Lancet Glob Health. 2018;6:e555-e567.

6. Lin X, Jia M, Zhou X, et al. The diagnostic performance of automated versus handheld breast ultrasound and mammography in symptomatic outpatient women: a multicenter, cross-sectional study in China. Eur Radiol. 2021;31:947-957.

7. Berg WA, Blume JD, Cormack JB, et al. Combined screening with ultrasound and mammography vs mammography alone in women at elevated risk of breast cancer. JAMA. 2008;299:2151-63.

8. Shen $\mathrm{S}, \mathrm{Zhou} \mathrm{Y}, \mathrm{Xu} \mathrm{Y}$, et al. A multi-centre randomised trial comparing ultrasound vs mammography for screening breast cancer in high-risk Chinese women. Br J Cancer. 2015;112: 998-1004.

9. Shao H, Li B, Zhang X, Xiong Z, Liu Y, Tang G. Comparison of the diagnostic efficiency for breast cancer in Chinese women using mammography, ultrasound, MRl, and different combinations of these imaging modalities. J Xray Sci Technol. 2013;21:283-92.

10. $\mathrm{Li} \mathrm{J}$, Wang $\mathrm{H}$, Wang $\mathrm{L}$, et al. The concordance in lesion detection and characteristics between the Anatomical Intelligence and conventional breast ultrasound Scan method. BMC Med Imaging. 2021;21:102.

11. Thigpen D, Kappler A, Brem R. The Role of Ultrasound in Screening Dense Breasts-A Review of the Literature and Practical Solutions for Implementation. Diagnostics (Basel). 2018;8:20.

12. Berg WA, Bandos Al, Mendelson EB, Lehrer D, Jong RA, Pisano ED. Ultrasound as the Primary Screening Test for Breast Cancer: Analysis From ACRIN 6666. J Natl Cancer Inst. 2015;108:djv367.

13. Uematsu T. Ultrasonographic findings of missed breast cancer: pitfalls and pearls. Breast Cancer. 2014;21:10-9.

14. Huang CS, Yang YW, Chen RT, et al. Whole-Breast Ultrasound for Breast Screening and Archiving. Ultrasound Med Biol. 2017;43:926-933.

15. Bosch AM, Kessels AG, Beets GL, et al. Interexamination variation of whole breast ultrasound. Br J Radiol. 2003;76:328-31.

16. Šroubek F, Bartoš M, Schier J, et al. A computer-assisted system for handheld whole-breast ultrasonography. Int J Comput Assist Radiol Surg. 2019;14:509-516.

17. Jiang WW, Li C, Li AH, Zheng YP. Clinical Evaluation of a 3-D Automatic Annotation Method for Breast Ultrasound Imaging. Ultrasound Med Biol. 2016;42:870-81. 
18. Baker JA, Soo MS. Breast US: assessment of technical quality and image interpretation. Radiology. 2002;223:229-38.

19. Chang JF, Huang CS, Chang RF. Automated whole breast segmentation for hand-held ultrasound with position information: Application to breast density estimation. Comput Methods Programs Biomed. 2020;197:105727.19

20. Niu L, Bao L, Zhu L, et al. Diagnostic Performance of Automated Breast Ultrasound in Differentiating Benign and Malignant Breast Masses in Asymptomatic Women: A Comparison Study With Handheld Ultrasound. J Ultrasound Med. 2019;38:2871-2880.

21. Zhang X, Chen J, Zhou Y, et al. Diagnostic value of an automated breast volume scanner compared with a hand-held ultrasound: a meta-analysis. Gland Surg. 2019;8:698-711.

22. Berg WA, Blume JD, Cormack JB, Mendelson EB, Madsen EL; ACRIN 6666 Investigators. Lesion detection and characterization in a breast US phantom: results of the ACRIN 6666 Investigators. Radiology. 2006;239:693-702.

23. Bozzini A, Renne G, Meneghetti L, et al. Sensitivity of imaging for multifocal-multicentric breast carcinoma. BMC Cancer. 2008;8:275.

24. Park JM, Yang L, Laroia A, Franken EA Jr, Fajardo LL. Missed and/or misinterpreted lesions in breast ultrasound: reasons and solutions. Can Assoc Radiol J. 2011;62:41-9.

25. Berg WA, Blume JD, Cormack JB, Mendelson EB. Operator dependence of physician-performed whole-breast US: lesion detection and characterization. Radiology. 2006;241:355-65.

26. Berg WA. Sonographically depicted breast clustered microcysts: is follow-up appropriate? AJR Am J Roentgenol 2005;185:952-9.

\section{Figures}




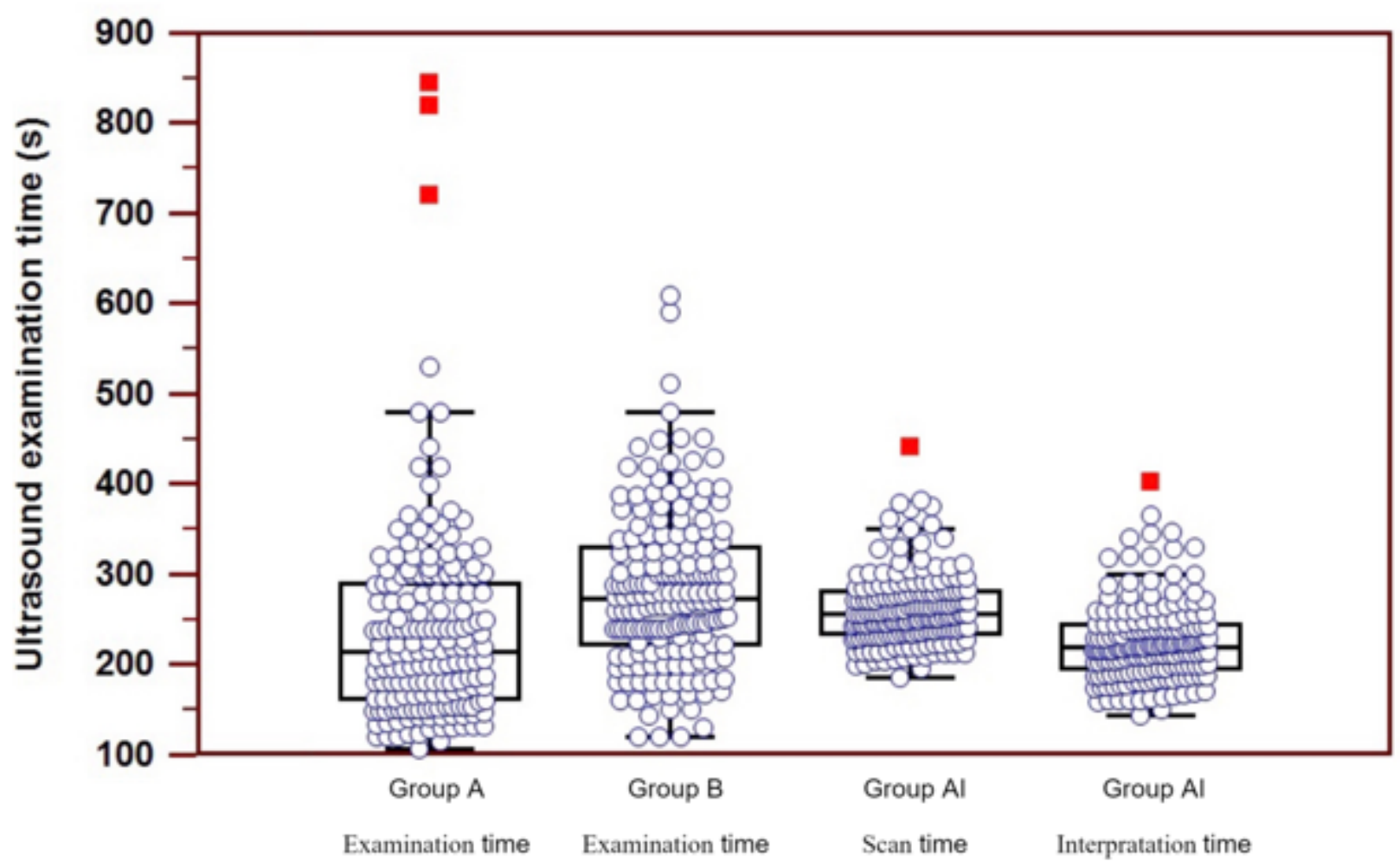

Figure 1

Al-Breast and HHUS examination time quadrants. Red squares in the plot indicate that the value is larger than the upper quartile and is 3 times the quartile difference. 